\title{
Facial morphospace: a clinical quantitative analysis of the three-dimensional face in patients with cleft lip and palate
}

\author{
Chihiro Tanikawa \\ Department of Orthodontics and Dentofacial Orthopedics, Graduate School of Dentistry, Osaka University, Osaka 565-0871, \\ Japan.
}

Correspondence to: Assoc Prof./Dr. Chihiro Tanikawa, Department of Orthodontics and Dentofacial Orthopedics, Graduate School of Dentistry, Osaka University, Osaka 565-0871, Japan. E-mail: ctanika@dent.osaka-u.ac.jp

How to cite this article: Tanikawa C. Facial morphospace: a clinical quantitative analysis of the three-dimensional face in patients with cleft lip and palate. Plast Aesthet Res 2020;7:48. http://dx.doi.org/10.20517/2347-9264.2020.136

Received: 15 Jun 2020 First Decision: 27 Jul 2020 Revised: 5 Aug 2020 Accepted: 20 Aug 2020 Published: 12 Sep 2020

Academic Editor: Carroll Ann Trotman Copy Editor: Cai-Hong Wang Production Editor: Jing Yu

\begin{abstract}
Aim: The aims were: (1) to examine the clinical application of a geometric morphometric method (GMM) that quantifies the three-dimensional (3D) configuration of the facial soft tissues in patients with a repaired unilateral cleft lip and palate (UCLP); and (2) to determine the morphological characteristics that distinguish between noncleft participants and patients with UCLP.
\end{abstract}

Methods: 3D facial images at rest were recorded from Japanese patients with a repaired UCLP (Cleft group; $n=$ 60) and healthy adults featuring a straight type facial profile with normal occlusion (Control group; $n=200$ ) using $3 \mathrm{D}$ photogrammetric cameras. For each participant, wire mesh fitting was conducted based on the assignment of landmarks to each 3D facial image. This method generated landmark-based GMM models consisting of 6017 nodes on the fitted wire mesh. For each node, the mean and standard deviation were determined in the Control group and were used as the normative range of the faces. With this normative range, the Z-scores before and after surgery were evaluated for patients with UCLP who underwent orthognathic bimaxillary surgery. Further, the morphological characteristics of the Cleft group were evaluated using a principal component (PC) regression analysis that distinguished between two subject groups. In addition, K-means clustering analysis and MANOVA were used to examine the morphological variation of the Cleft group.

Results: A patient with UCLP was evaluated with the system. After surgery, the normal area increased by $8 \%-20 \%$ on all axes, which means that the surgery was effective for normalizing the patient's face. However, even after

\footnotetext{
(@) $(0$

(C) The Author(s) 2020. Open Access This article is licensed under a Creative Commons Attribution 4.0 International License (https://creativecommons.org/licenses/by/4.0/), which permits unrestricted use, sharing, adaptation, distribution and reproduction in any medium or format, for any purpose, even commercially, as long as you give appropriate credit to the original author(s) and the source, provide a link to the Creative Commons license, and indicate if changes were made.
}

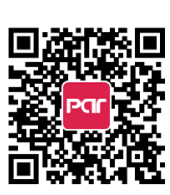


surgery, the protrusion of the lower lip and asymmetry remained. Nine PCs were extracted, and seven PCs were selected for the regression model to discriminate two subject groups, e.g., midfacial retrusion, nasal bump, and chin protrusion. The MANOVA also revealed significant differences between both the Cleft and Control groups and the sex subgroups, and the effects of cleft on the facial morphology was found to be related to sex (all, $P<$ 0.01).

Conclusion: The clinical application of GMM was confirmed to be effective. GMM detected variations of the Cleft group and morphological characteristics. GMM is considered to be a powerful tool to quantitatively evaluate faces in clinics.

Keywords: Cleft lip and palate, face, geometric morphometric analysis, three-dimensional analysis

\section{INTRODUCTION}

The extent of recognition of an individual's own face by others exerts a great sociopsychological influence on that individual's sense of acceptance by his or her community. It is known that there are several facial deformities associated with congenital cleft lip after primary lip revision surgery. It is thus crucial for patients with cleft lip and/or palate to normalize their facial morphology with primary repair and any subsequent revision surgery. The extent and type of these facial deformities varies among patients due to the extent of the original abnormality and any prior surgeries performed ${ }^{[1-3]}$. Several experts have recommended using subjective checklists to provide assessments of the extent and type of facial abnormalities ${ }^{[4,5]}$; however, this could result in bias. The development of a systematic method for the evaluation and classification of the morphological traits of the cleft facial shape could greatly facilitate the surgical/orthodontic diagnosis and the design of treatment plans for the optimization of treatment outcomes.

The analysis of the form of the nose and lip is important for revision surgery and many attempts have been made to establish systematic analytical methods. Conventional photographs have been employed in some studies ${ }^{[3,6,7]}$. Several three-dimensional (3D) analysis techniques, including direct anthropometry of the human face ${ }^{[8,9]}$, stereophotogrammetry ${ }^{[10,11]}$, laser scanning, gypsum cast ${ }^{[12-14]}$, and computer tomography ${ }^{[15]}$, have been employed to assess the facial morphology of patients with cleft lip. Because these analyses are primarily conducted with the use of linear and/or angular measurements between/among landmarks, the detailed morphological characteristics of the entire facial surface forms are not considered. There have also been a few attempts to extract detailed morphological characteristics of the entire facial surface forms using the slice lines. In these studies, traced nasal lines in an axial view were assessed using a curve fitting method that provides the extent of asymmetry ${ }^{[12]}$, and an automatically extracted nostril shape was assessed $^{[16]}$. These studies succeeded in describing the morphological characteristics of the nasal surface; they mainly attempted to describe the nasal forms in an axial view and the nostril shapes before and after surgery. A 3D quantitative analysis of the lip was also reported, in which the morphological traits of the lip surfaces were measured from the frontal and lateral views ${ }^{[17]}$. However, thus far, few studies have reported the $3 \mathrm{D}$ holistic analysis of the entire facial form from all three views (i.e., frontal, lateral, and axial views).

With recent computation advances, landmark-based geometric morphometric methods (GMMs) have recently emerged that-together with conventional measurements in medicine-have revealed some statistical variation in the shape and size of target objects (phenotypic variation). In developmental biology, GMMs use homologous landmarks, which can be defined as precise locations on biological specimens that hold some functional, structural, developmental, or evolutionary significance, and which are directly comparable between specimens $^{[18]}$. GMMs have four types of landmarks: Type I landmarks can be clearly defined on a 
structure (e.g., the corner of the mouth); Type II landmarks are more ambiguous and usually describe the maxima of curvature (e.g., nasal tip); Type III landmarks are geometric constructions generated from lines or Type I and/or II landmarks (e.g., midpoint of the right and left eyes); and semi-landmarks represent surfaces or curves between landmarks ${ }^{[18]}$. A key concept of GMM is based on the fact that morphology can be mapped systematically, often within a "morphospace", with the use of these landmarks. Morphospaces are maps that show how shapes are defined by quantitative traits. GMMs rely on the superimposition of landmark coordinate data $\mathrm{r}$ to place individuals in a common morphospace.

Recently, the clinical application of a GMM-a novel 3D quantitative analysis method-in the quantification and visualization of the $3 \mathrm{D}$ configuration of the facial soft tissues was reported ${ }^{[19]}$. $3 \mathrm{D}$ faces were fitted with mathematical wire meshes based on 26 landmarks, and the nodes of the fitted meshes were used as semilandmarks. All faces were superimposed based on the landmarks and were statistically analyzed in the facial morphospace. The analysis included the average range of faces with regard to sex, age, and race, and the method compared a patient's face with this normal range. This enabled us to understand the patient's static facial form characteristics quantitatively and instantaneously. In their report, the applicability of the system to three cases, namely one case each of Class II malocclusion, Class III malocclusion, and jaw deviation, was reported; however, the applicability of this system to the cleft facial shape remains unclear.

Thus, the objectives of this study were as follows: (1) to examine whether the previously published softtissue evaluation methods developed based on $\mathrm{GMMs}^{[19]}$ could be applied to patients with a cleft lip before or after treatment; (2) to determine soft-tissue morphological characteristics that distinguish noncleft participants from patients with a cleft lip; and (3) to examine the variations of cleft facial shapes. Furthermore, we discuss the clinical application of the GMM based on the results of Objectives (1)-(3).

\section{METHODS}

The study was approved by the ethics committee for medical research at Osaka University Dental Hospital (ID: H25-E37-1). An informed consent to participate in the study was obtained from all participants.

\section{Systems}

A system for evaluating facial morphology that was developed in a previous study ${ }^{[19]}$ was employed in the present study. The overview of the system is shown in Figure 1. The system was divided into three parts: (1) mesh fitting on the 3D facial picture; (2) development of the normative ranges of faces; and (3) evaluation of the new face with the normative face. In the present study, the normative range of the face was defined as the average and \pm 1 standard deviation ( $\pm 1 \mathrm{SD}$ ), and the patient data were compared to the normative range of faces. To develop the normative ranges, 200 participants (female, $n=100$; male, $n=100$ ) between 18 and 35 years of age were recruited from the students and faculty of Osaka University in Japan. For the detailed inclusion criteria, please see the previous study ${ }^{[19]}$. Then, the normative faces, including the mean coordinate values and the standard deviation for each sex group, were used to evaluate new patients. The equations were as follows:

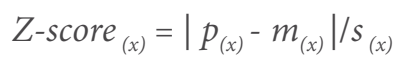

Z-score $_{(y)}=-\left(p_{(y)}-m_{(y)}\right) / s_{(y)}$

Z-score $_{(z)}=\left(p_{(z)}-m_{(z)}\right) / s_{(z)}$

where $p_{(x)}, p_{(y)}$, and $p_{(z)}$ indicate the coordinate values of each image of the sample patients; $m_{(x)}, m_{(y)}$, and $m_{(z)}$ indicate the average coordinate values of the control group; and $s_{(x)}, s_{(y)}$, and $s_{(z)}$ indicate the standard deviation of the coordinate values of the control group in the $x^{-}, y^{-}$, and $z$-directions, respectively. To visualize the results, the $\mathrm{Z}$-scores were visualized as color values. For a detailed description, please see the previous study $^{[19]}$. 

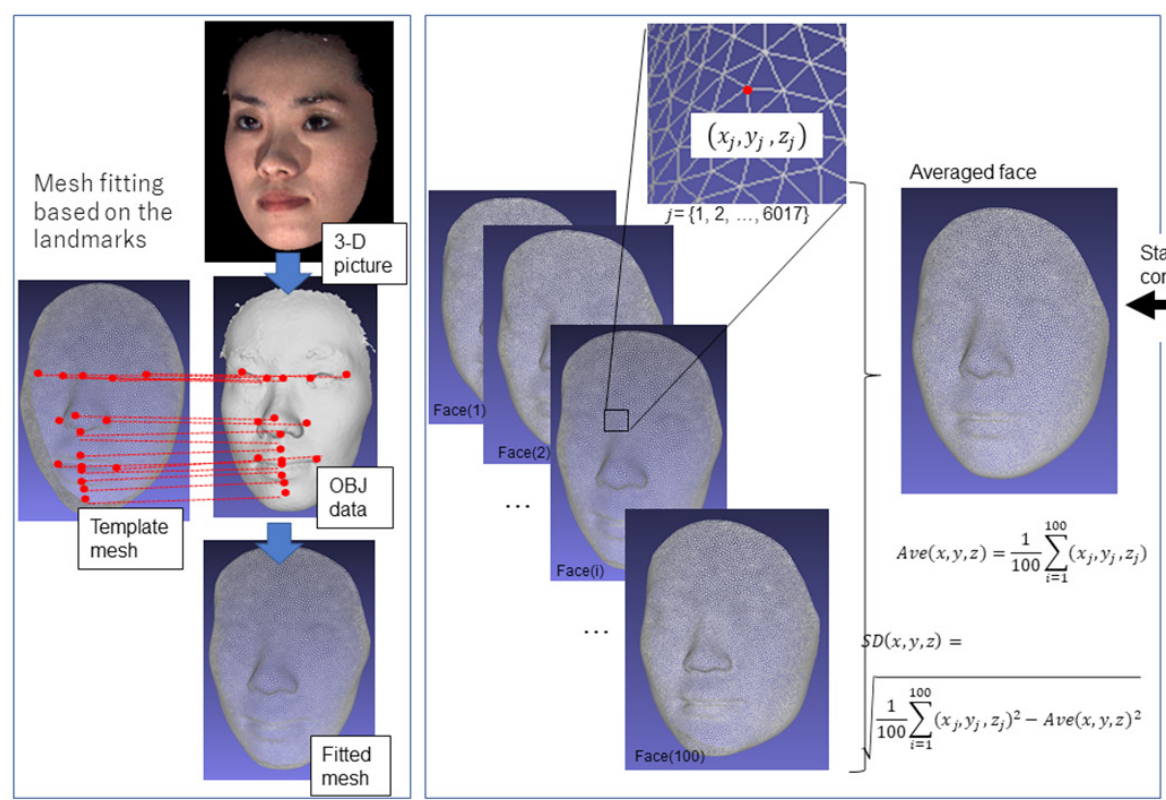

the normative face

Figure 1. A schematic illustration of the wire mesh fitting (Step 0), the generation of averaged faces (Step 1), and their statistical analysis (Step 2)

\section{Samples}

Application of the system to patients with unilateral cleft lip and palate

To demonstrate the applicability of the system, one Japanese patient with unilateral cleft lip and palate (UCLP) (example case) was selected from the patients who visited our department to undergo orthognathic bimaxillary surgery (Le Fort I maxillary surgery and bilateral sagittal split osteotomy, Figure 2). The details of the treatment have been described elsewhere ${ }^{[20]}$.

\section{Morphological characteristics of the faces of patients with UCLP}

Japanese patients with a repaired UCLP (Cleft group, $n=60$; mean age, $22.0 \pm 5.0$ years; male, $n=36$; female, $n=24$ ) and healthy adults with a straight-type facial profile with normal occlusion (Control group: $n=200$; mean age, $25.4 \pm 5.3$ years; male, $n=100$; female, $n=100$ ) were enrolled in the present study. The Control group samples were the same as those used in the previous study ${ }^{[21]}$. The inclusion criteria for the Cleft group were as follows: UCLP, patients who visited the orthodontic department, Osaka University Dental Hospital, during 2011-2015; age 15-37 years; positive overjet (i.e., most patients were in the posttreatment "retention" period); no facial paralysis; body mass index, 18.50-24.99; and no maxillofacial plastic surgery in the past six months. A written informed consent form was distributed to and signed by all participants.

\section{Data acquisition}

The subjects were asked to sit on a fixed chair with a natural head position without head support. Facial images at rest were recorded once with a 3D image capturing device (3-DMDcranial System, 3-DMD, Atlanta, GA, USA). This data acquisition was conducted once for each patient. For an example case to show the pre- and post-treatment changes, 3D images were acquired three months before and six months after treatment. 

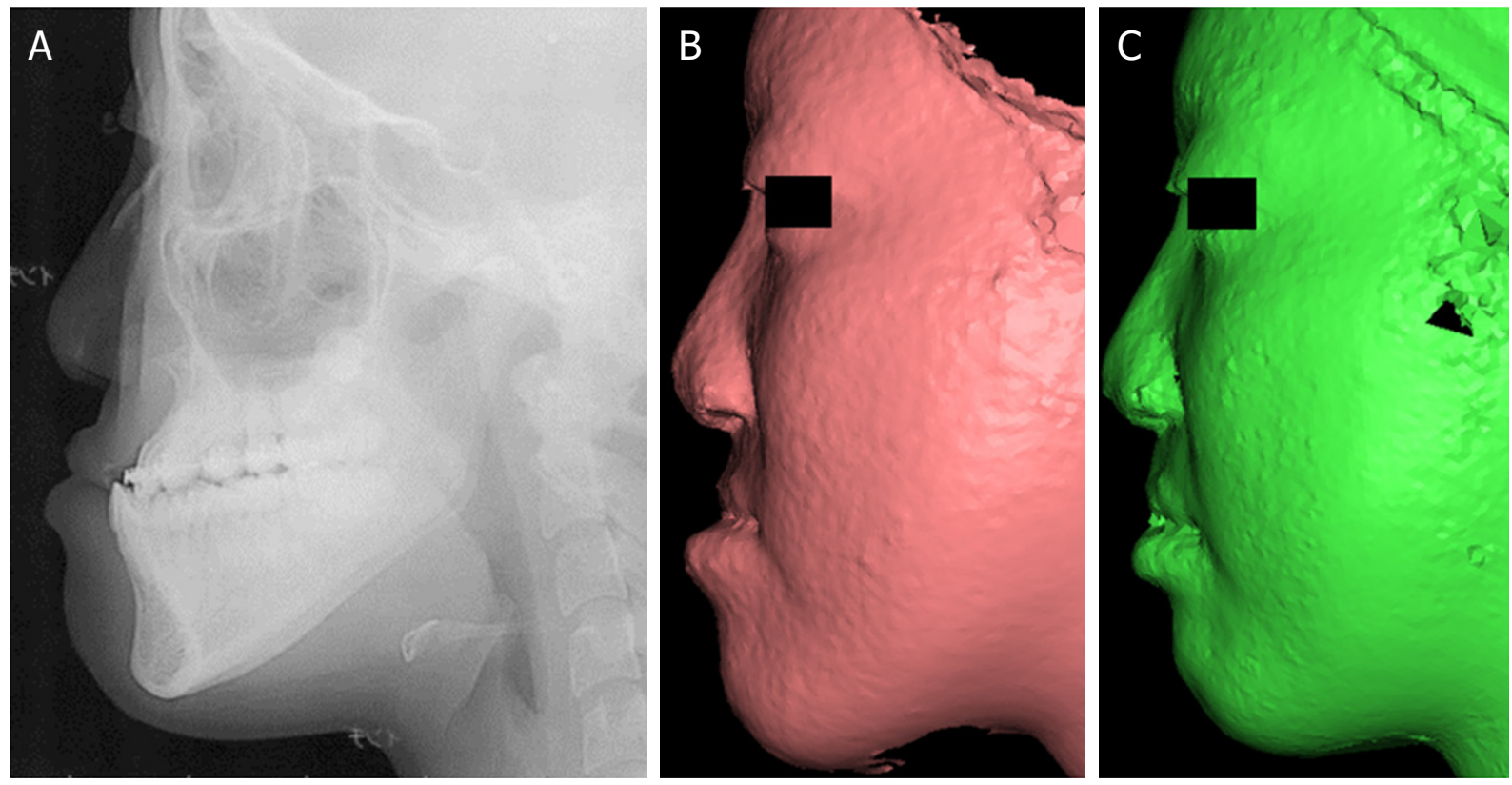

Figure 2. Cephalogram and 3D facial images of a patient with cleft lip and palate: cephalogram (A); 3D image pre-treatment (B); and 3D image post-treatment (C). These images were used for applicability of the system proposed in the previous study ${ }^{[19]}$

\section{Data processing}

\section{Landmark identification}

Each 3D facial image was displayed on a monitor, and the positions of 18 landmarks [nasion, pronasale, subnasale, labiale superious, stomion, labiale inferious, submentale, pogonion, porion (right and left), exocanthion (right and left), endocanthion (right and left), alar curvature (right and left), and cheilion (right and left) ${ }^{[8]}$ ] were identified and digitized using a commercial software program (Face Rugle, Medic Engineering Co., Kyoto, Japan). Facial images were standardized with a common coordinate system [Figure 3] ${ }^{[21]}$.

\section{Wire mesh fitting}

For each participant, landmark-based GMM analysis ${ }^{[22]}$ was performed using HBM software (National Institute of Advanced Industrial Science and Technology, Japan). This analysis generated a fitted mesh consisting of a set of 6017 points as nodes. The arithmetic means $m_{(x)}, m_{(y)}$, and $m_{(z)}$ and standard deviations $s_{(x)}, s_{(y)}$, and $s_{(z)}$ of the points were computed, and $m_{(x)} \pm s_{(x)}, m_{(y)} \pm s_{(y)}$, and $m_{(z)} \pm s_{(z)}$ were used as the normative range for each point in the X-, Y-, and Z-directions, respectively.

\section{Quantitative facial evaluation before and after treatment}

For the example case, the system was applied to exemplify the system results. Surface areas showing -1 SD $<$ Z-score $<1$ SD were defined as the normal area. The percentages of the surface before and after treatment were evaluated in each axis and presented as radar charts.

\section{Morphological differences between Cleft and Control groups}

For the Cleft and Control groups, to determine morphological characteristics of the facial soft tissue surface, the coordinate values of each node of the wire mesh on the facial surface on the $\mathrm{x}, \mathrm{y}$, and $\mathrm{z}$ axes were statistically analyzed. A two-sample $t$-test was performed to compare each axis in the two subject groups. To visualize the differences between the two subject groups, the results were represented as a color map showing the $P$-values for the comparison between the two subject groups (hereafter referred to as the significance probability map) and a color map representing the differences between two subject groups (hereafter referred to as the distance map) ${ }^{[21,22]}$. $P$ values of $<0.05$ were considered to indicate statistical significance. 


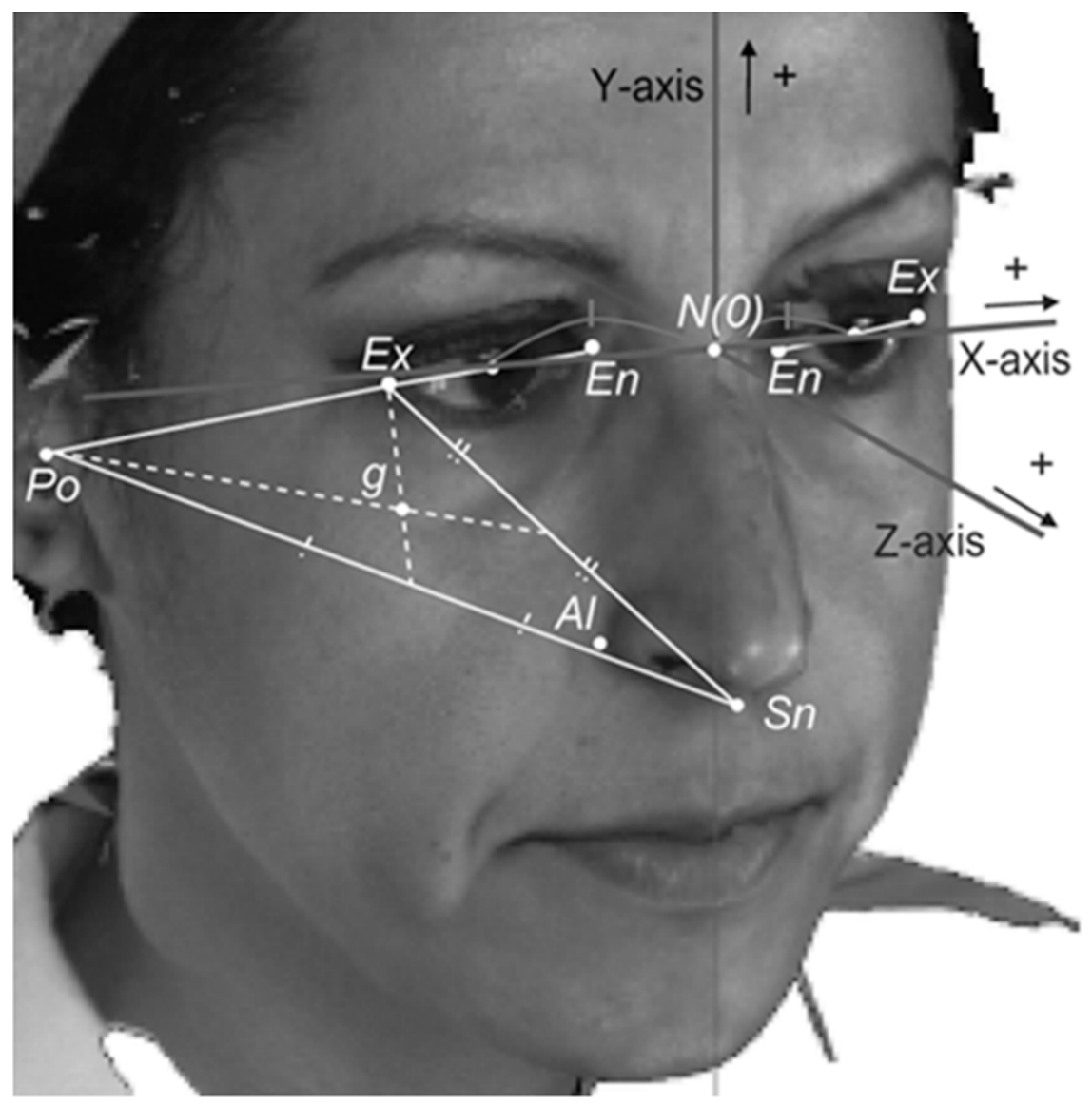

Figure 3. The coordinate system ${ }^{[19]}$. The $3 \mathrm{D}$ coordinate system. The nasion $(\mathrm{N})$ was defined as the origin $(\mathrm{O})$. The sagittal plane was defined as the plane passing through the origin and perpendicular to the line through the midpoint of the right exocanthion (Ex) and endocanthion (En) and the midpoint of the left Ex and En. The axial plane was defined as the plane passing through the origin and parallel to the line connecting the porion and the geometric center ( $g$ ) of the porion (Po), subnasale ( $\mathrm{Sn}$ ), and Ex on the image projected onto the sagittal reference plane. The coronal plane was defined as a plane passing through the origin and perpendicular to both the axial and sagittal planes (cited from Tanikawa et al. ${ }^{[21]}$ ). Images of the participants with a right unilateral cleft lip were "mirrored" to left side to simulate a left unilateral cleft lip

\section{Morphological variation of the Cleft group}

To examine the variance in each group, each sex, and their interactions, the following two analyses were also conducted. We first performed a principal component analysis for the 6017 coordinates of each point on the facial surface in the $\mathrm{x}, \mathrm{y}$, and $\mathrm{z}$ axes to reduce dimensionality. Significant principal components (PCs) were determined by a scree plot analysis ( $90 \%$ was used as the cut off value). Significant PCs were entered into a regression model that decimated the Control and Cleft groups $(P<0.01)$. PCs were also entered into a MANOVA to test for significance of factors (i.e., cleft/non-cleft and sex). Then, to examine the patterns of the face and variation of the Cleft group, the patients in the Cleft group were categorized based on the similarities in the morphospace constructed above PCs using a clustering method (k-means and Elbow method).

\section{RESULTS}

\section{Quantitative facial evaluation before and after treatment}

Figure 4 shows the results of the application of the system to a patient with UCLP before and after treatment. The percentage of the normal area $(-1 \mathrm{SD}<Z$-score $<1 \mathrm{SD})$ on each axis is shown in Figure 5 for each facial region before and after treatment. In total, the percentage of the normal area was increased from $20 \%$ to $40 \%$ on the $\mathrm{x}$-axis (transverse direction); from $52 \%$ to $59 \%$ on the $\mathrm{y}$-axis (vertical direction); 
Transverse (X-axis)
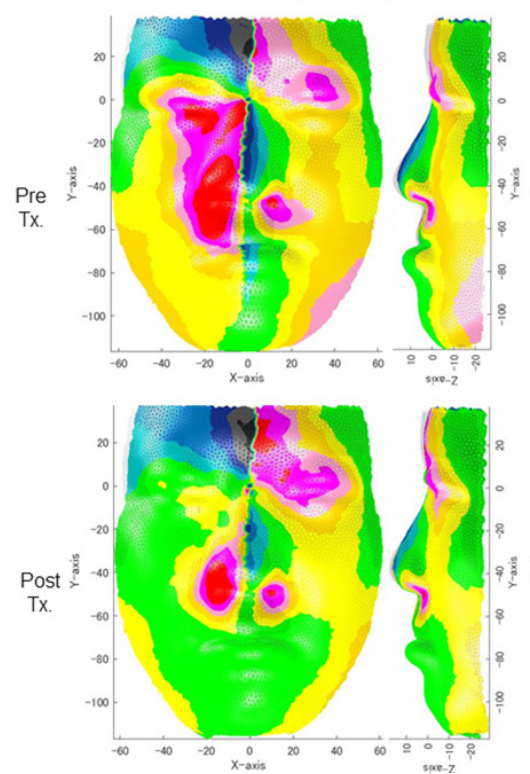

Vertical (Y-axis)
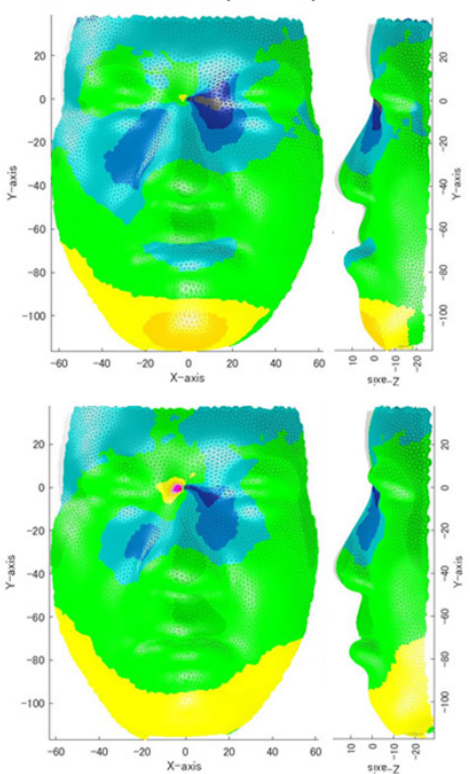

Antero-posterior (Z-axis)
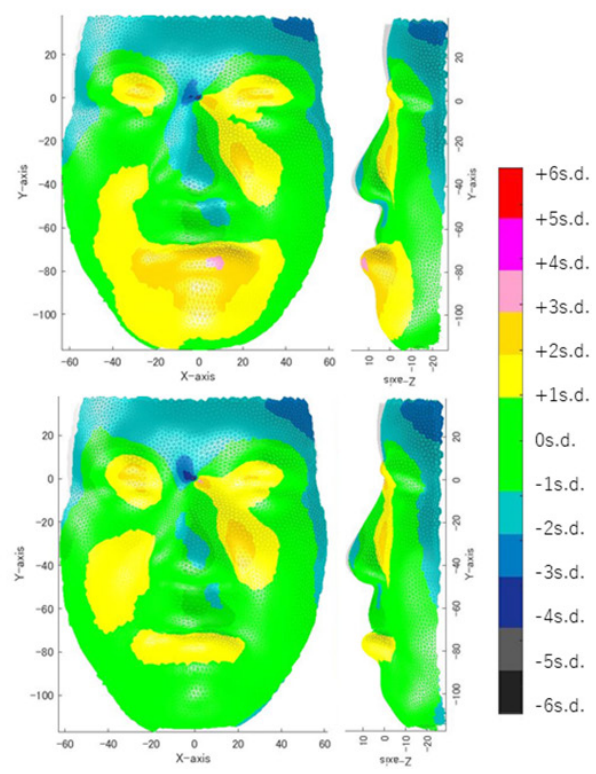

Figure 4. Verification of the system using a cleft case. Grey profile indicates an average face: (top) pre-surgery; and (bottom) postsurgery. (left) The standardized X-values of the 3D face when compared with the normative mean (transverse direction). Red, pink, light yellow, and deep yellow represent the point clouds located outwards when compared with the normative mean; light blue, deep blue, grey, and black indicate the points located inwards (close to the medial line). Green represents the range within \pm 1 SD. The left column indicates the standardized $Z$-value (transverse direction). Before treatment, the right nasal alar and nasal tip were deviated to the right side by more than $6 \mathrm{SD}$. After treatment, the area showing more than 1 SD was decreased; however, the right nasal alar was still deviated by 6 SD (for the axial view, please see Figure 6). (middle) The standardized Y-value (vertical direction). Red, pink, light yellow, and deep yellow indicate the corresponding point clouds located inferiorly, whereas light blue, deep blue, grey, and black indicate the points located superiorly when compared with the normative mean. In this patient, before treatment, the lower lip was located superiorly by $1 \mathrm{SD}$, and the chin was located inferiorly by more than $2 \mathrm{SD}$ and showed greater facial height. After treatment, the lower lip was located within the normal range, indicating that the lower lip had moved downwards after maxillary advancement and mandibular set-back movement due to the surgery. (right) The standardized $Z$-value (antero-posterior direction). Red, pink, light yellow, and deep yellow indicate the corresponding point clouds located anteriorly, whereas light blue, deep blue, grey, and black indicate the points that were located posteriorly when they were compared to the normative mean. In this patient, before treatment, the nasal dorsum and left upper lip (repaired cleft site) retruded by more than 1SD, while the chin protruded by more than 3 SD. After treatment, the chin showed normal antero-posterior position while the lower lip showed slight retrusion by more than $1 \mathrm{SD}$. The area of the retrusion in the nasal dorsum and upper lip was also decreased

Transverse (X-axis)

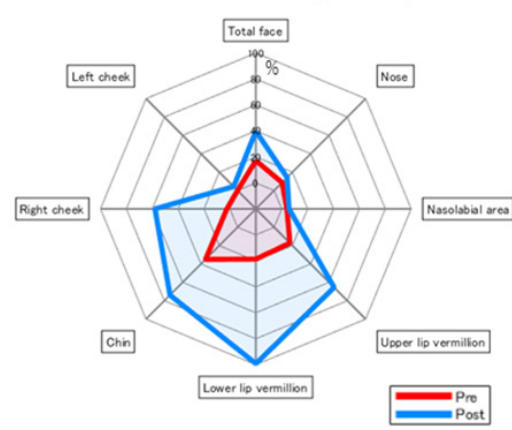

Vertical (Y-axis)

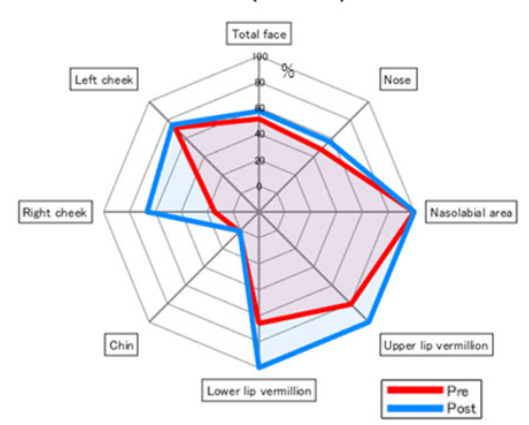

Antero-posterior (Z-axis)

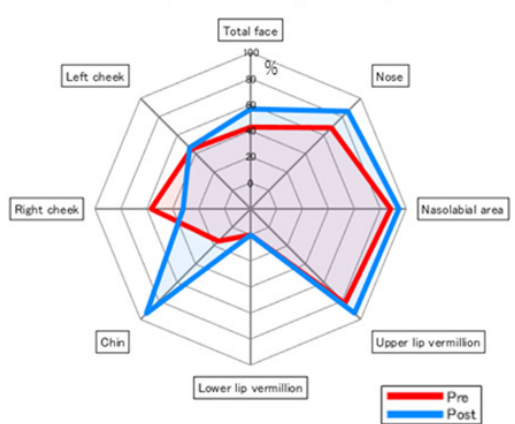

Figure 5. Radar chart of the normal area for the example case with unilateral cleft lip and palate [Figure 2]. Surface areas showing -1 SD $<$ Z-score < 1SD were defined as the normal area. The percentage of the surface before (red) and after treatment (blue) was evaluated on: $x$-axis (left); $y$-axis (middle); and z-axis (right)

and from $41 \%$ to $59 \%$ on the $\mathrm{z}$-axis (antero-posterior direction). In detail, the figures for the $\mathrm{x}$-axis show that before surgery there was greater asymmetry in the cheeks, the nose, nasolabial region, upper lip, 


\section{Transverse (X-axis)}
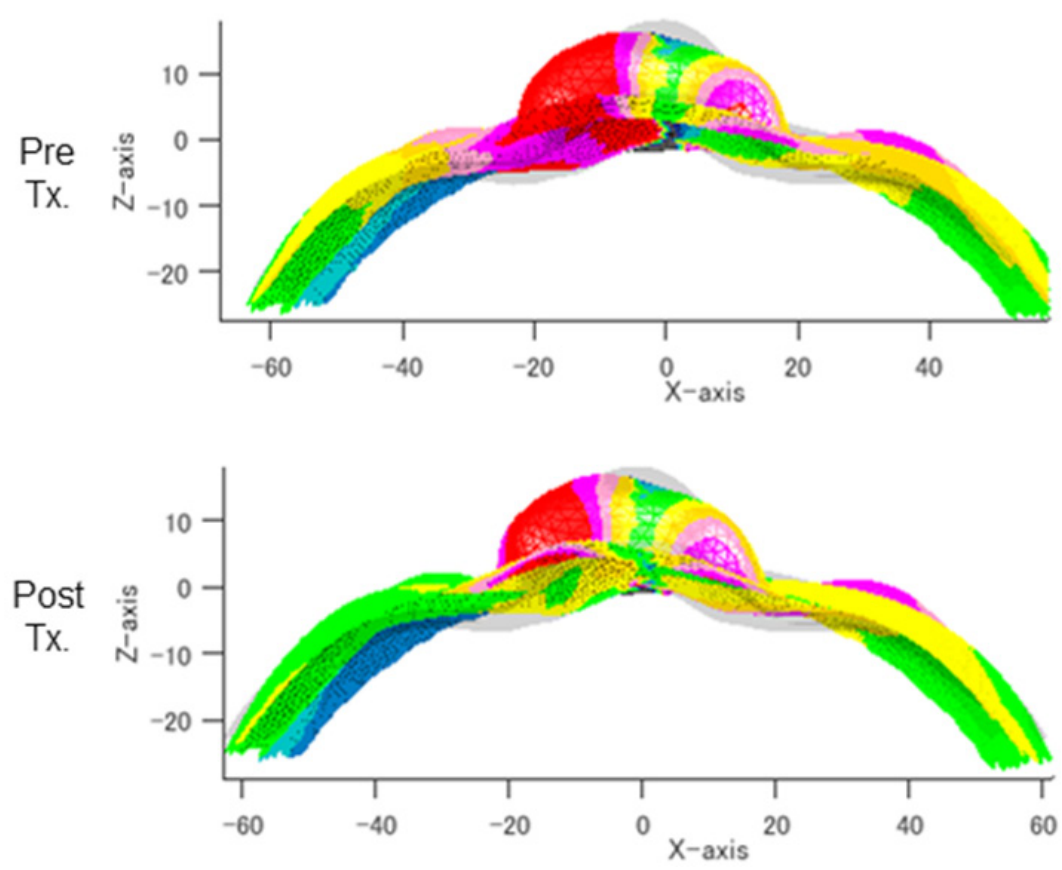

Figure 6. Axial view of the standardized $X$-values of the 3D nasal dorsum in comparison to the normative mean (transverse direction): (top) pre-surgery; and (bottom) post-surgery. Grey indicates the averaged face of the Control group (female; $n=100$ ). Red, pink, light yellow, and deep yellow represent the point clouds located outwards when compared with the normative mean; light blue, deep blue, grey, and black indicate the points located inwards (close to the medial line). Green represents the range within \pm 1 SD. Before treatment, the right nasal base was deviated to the right side by more than 6 SD before and after treatment. The left nasal base was also deviated to the left side by more than 5 SD before and after treatment. These results indicate that the width of the nose was greater in comparison to the normative range, which was not corrected, even after bimaxillary surgery

lower lip, and chin. After surgery, the asymmetry in the upper and lower lip vermilion (the normal area changed from $19 \%$ to $84 \%$ and from $20 \%$ to $100 \%$, respectively; Figure 5, left) and the right cheek improved remarkably (from $20 \%$ to $60 \%$ ); however, the nose and nasolabial area and left cheek showed deviation to the right (x-axis), which is clearly observed in Figure 6 showing the axial view. In the z-axis, the figures also show that, before surgery, the patient had a retruded area corresponding the cleft scar $(-1 \mathrm{SD})$ and a protruded chin $(+1$ to $+2 \mathrm{SD})$. After surgery, the retrusion of the chin (normal area was changed from $18 \%$ to $98 \%$; Figure 5, right) and lower lip improved remarkably, yet maintained a mean of -1 SD of the normative face for the lower lip (z-axis). As for the vertical direction, the upper lip vermillion was displaced downward (y-axis) into the normative range after surgery (from 90\% to 100\%; Figure 5, middle), while the greater facial height remained after surgery (from $20 \%$ to $20 \%$; Figure 5, middle).

\section{Morphological differences between the Cleft and Control groups}

Figures 7 and 8 show the average differences between the Control and Cleft groups and the significance probability map of the X-, Y-, and Z-values, respectively.

In the transverse direction ( $\mathrm{x}$-axis), the Cleft group showed that significant widening of the nasal wall at the non-cleft side, and the nasal dorsum and nasal alar of the non-cleft side was deviated to the non-cleft side. The upper lip, lower lip, and chin were significantly deviated to the cleft side $(P \leq 0.001)$. This indicates that the nasal tip was more rounded on the non-cleft side. 

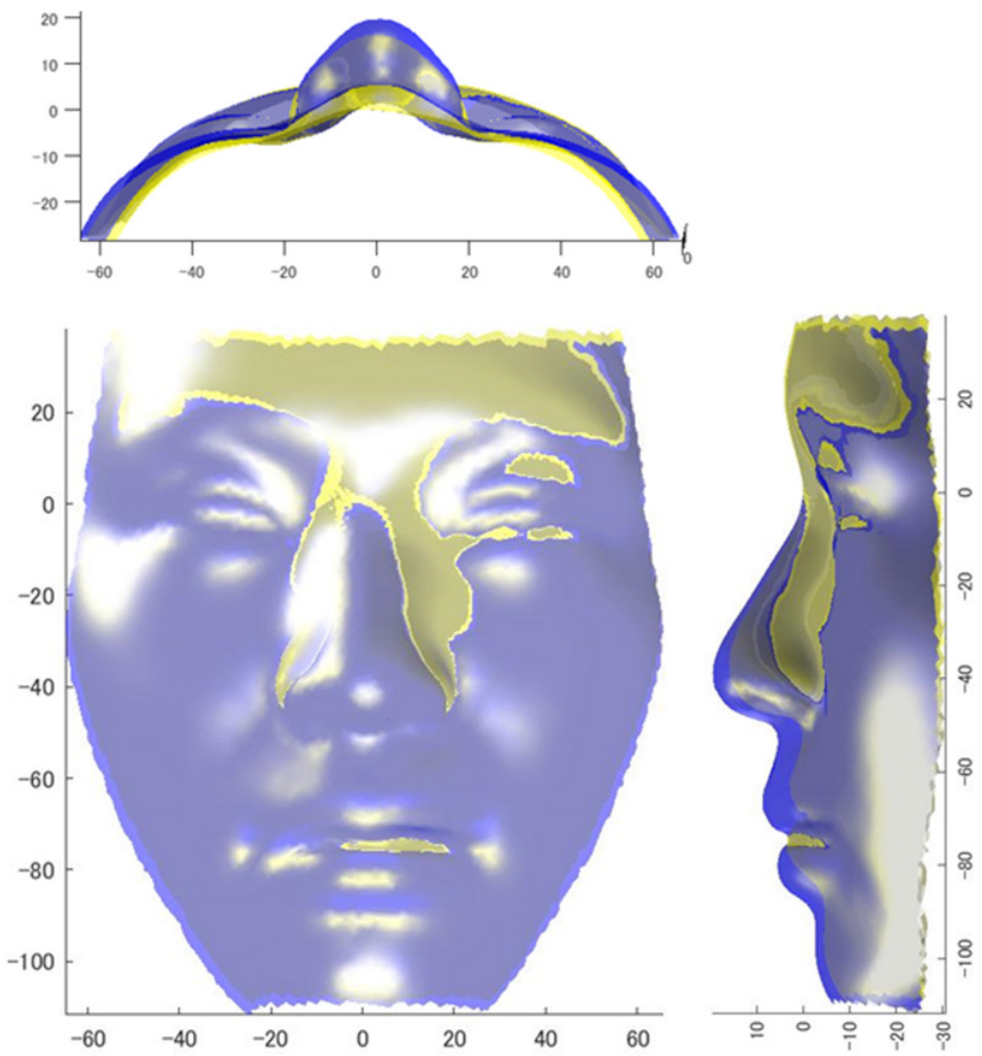

\section{Cleft \\ Control}

Figure 7. Average shape of the Cleft group (yellow) and Control group (blue). The statistical significance of the differences is shown in Figure 8
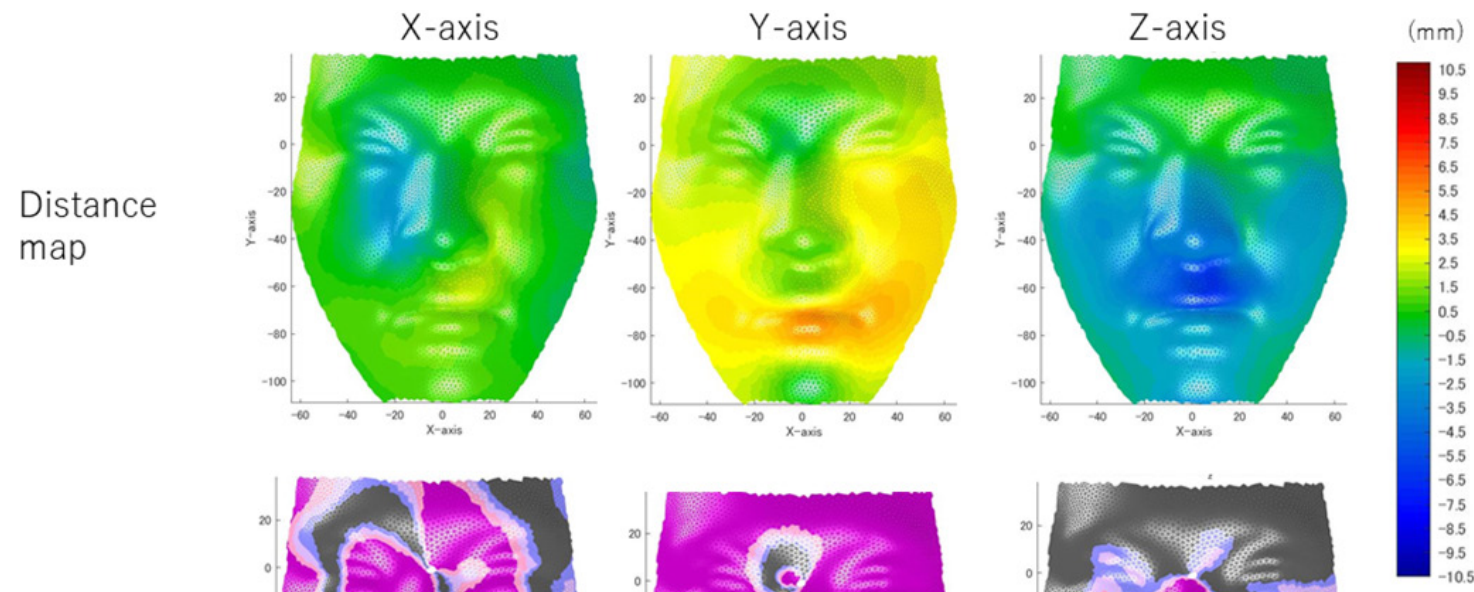

Significance probability map
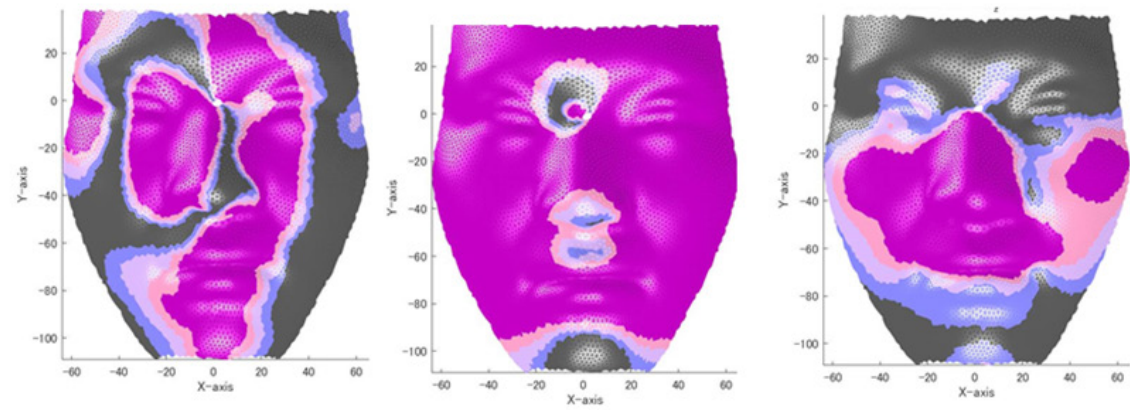

Figure 8. Distance maps (top) and significance probability maps (bottom) of the difference in the coordinate values of nodes of the fitted mesh between the Control and Cleft groups in three directions [horizontal (left); vertical (middle); and anteroposterior (right) directions]. For the significance probability maps, the colors designate: blue, $P \leq 0.05$; pale pink, $P \leq 0.01$; dark pink, $P \leq 0.001$; and purple, $P \leq 0.0001$. For the distance maps, red indicates that the difference in the coordinate values between two subject groups (Cleft group - Control group) is a positive value, whereas blue indicates that the difference in the coordinate values between the two subject groups (Cleft group - Control group) was a negative value 

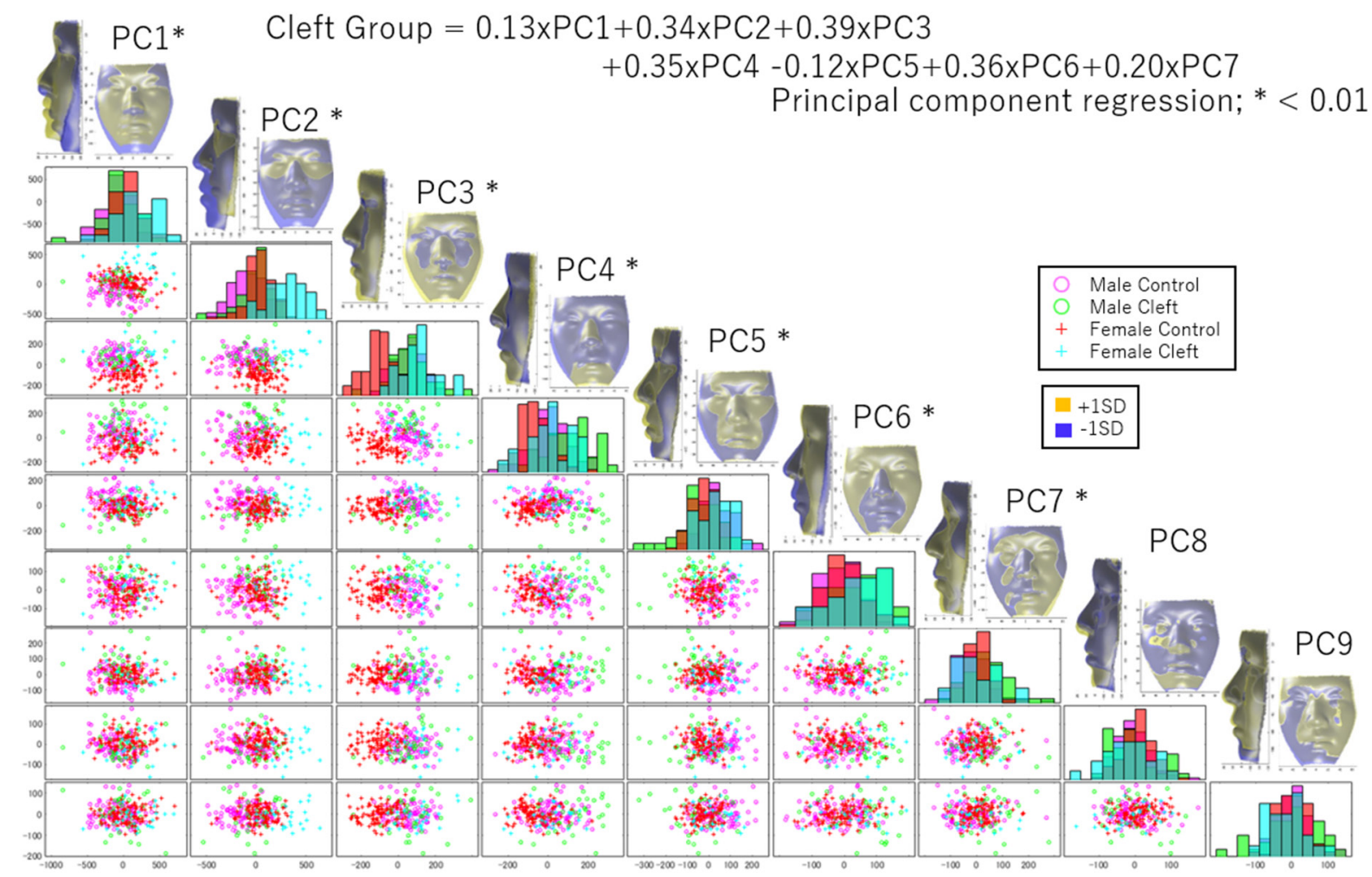

Figure 9. A scatter plot matrix of the principal component (PC) scores in the Cleft and Control groups with a histogram in the diagonal cells. PCs 1-9 explain $90.5 \%$ of the shape variation across samples. Pink denotes facial configurations in the male Control group (male); green denotes those in the male Cleft group; red denotes those in the female Control group; and cyan denotes those in the female Cleft group. The PCs are defined as +1 SD (yellow) and -1 SD (blue) in the top column. For example, the top column indicates that a +1 SD value of PC2 represents a smaller facial height, which was represented as yellow (1 SD). The histogram shows that the bimodal distribution and female patients in the Cleft group (cyan) showed a greater PC2 value, suggesting that the female Cleft group had a smaller facial height

In the vertical direction (y-axis), the position of the lower lip was significantly higher by approximately $4.5 \mathrm{~mm}$ in the Cleft group; however, the upper lip showed vertically normal position $(P \leq 0.001)$.

In the antero-posterior direction (z-axis), the Cleft group showed significant retrusion of the nose, cheek, upper lip, and lower lip in comparison to the Control group at rest, by approximately $2.5-4.5 \mathrm{~mm}(P \leq 0.001)$. On the other hand, the nasal alar at the cleft side showed no differences between two groups, indicating nasal alar at the cleft side was flattened.

\section{Morphological variation of the Cleft group}

The first nine significant PCs, which explained $90 \%$ of the sample's variance, were determined to be significant by a scree plot analysis [Figures 9 and 10]. Based on the principal component regression analysis, PC3 (weight $=0.39$ ), PC6 (0.36), PC4 (0.35), and PC2 (0.34) were extracted as important features to discriminate the Cleft and Control groups. Visualization of the between-group structure of the surface data revealed that $\mathrm{PC} 3$ was characterized by midfacial retrusion, well-developed nasal bone, and chin protrusion, and the Cleft group showed greater PC3 values $(P<0.001)$. PC6 was characterized by retrusion of the nose, upper lip, and lower lip, and the Cleft group showed a greater PC6 value $(P<0.000)$. PC4 was characterized by the retrusion of the cheek, eyes, and upper lip, and the Cleft group showed a greater PC4 value than the Control group. PC2 was characterized by a vertically short face. The Cleft group showed greater PC2 values, indicating that the patients in the Cleft group showed vertically shorter faces. 


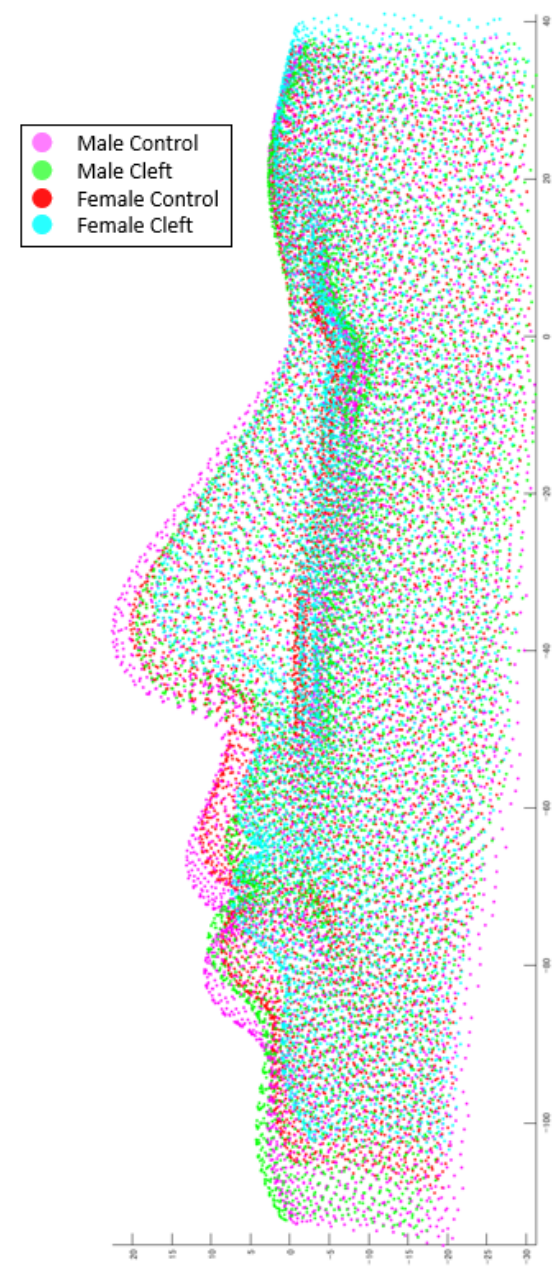

Figure 10. Superimposition of the averaged coordinate values of a male Control group patient (pink), a male Cleft group patient (green), a female Control group patient (red), and a female Cleft group patient (cyan)

MONOVA revealed that there were significant differences in the facial morphology in the Cleft and Control groups and sex the subgroups; and that the effects of cleft on the facial morphology were related to sex $[P<$ 0.01 , Table 1].

With these nine PCs, the optimal number of clusters was mathematically created. The Elbow method showed that the samples could be classified into four typical patterns of the Cleft group (i.e., codes) [Figures 11 and 12]. Using color maps, the differences among the codes were described in detail: midface retrusion with short mandibular height (Code 1), midface retrusion combined with mandibular protrusion with welldeveloped nasal bone (Code 2), smaller nasal height and retruded cheeks (Code 3), and severe mandibular protrusion (Code 4).

Table 2 shows the demographic data of patients in each code. Code 1 consists of female patients, while Codes 2-4 consist of male patients. It was difficult to compare the surgical techniques or orthodontic treatment, because the sample size in each attribution was small.

\section{DISCUSSION}

In the present research, we took advantage of GMM to propose a $3 \mathrm{D}$ quantitative analysis method for quantifying and visualizing the $3 \mathrm{D}$ configuration of the soft tissues of the face at rest. This method was 
Table 1. The multifactor analysis of variance of the surface-based model

\begin{tabular}{llllccc}
\hline & Df & Pillai & Approx F & Num Df & Den Df & Pr(> F) \\
\hline Cleft & 1 & 0.8149 & 108.756 & 10 & 247 & $<2.2 \mathrm{e}-16^{\star}$ \\
Sex & 1 & 0.6727 & 50.761 & 10 & 247 & $<2.2 \mathrm{e}-16^{\star}$ \\
Cleft: Sex & 1 & 0.1969 & 6.056 & 10 & 247 & $<3.13 \mathrm{e}-8^{\star}$ \\
Residuals & 256 & & & & & \\
\hline
\end{tabular}

$\star P<0.01$. Df: degrees of freedom; Pillai: Pillai's trace, which is a test statistic in the multifactor analysis of variance. This is a positive valued statistic ranging from 0 to 1. Increasing values means that effects are contributing more to the model; Approx F: the F statistic for the given predictor and test statistic; Num DF: the number of degrees of freedom in the model; Den Df: the number of degrees of freedom associated with the model errors; $\operatorname{Pr}(>\mathrm{F})$ : the $P$-value associated with the $\mathrm{F}$ statistic of a given effect and test statistic. The null hypothesis that a given predictor has no effect on either of the outcomes is evaluated with regard to this $P$-value

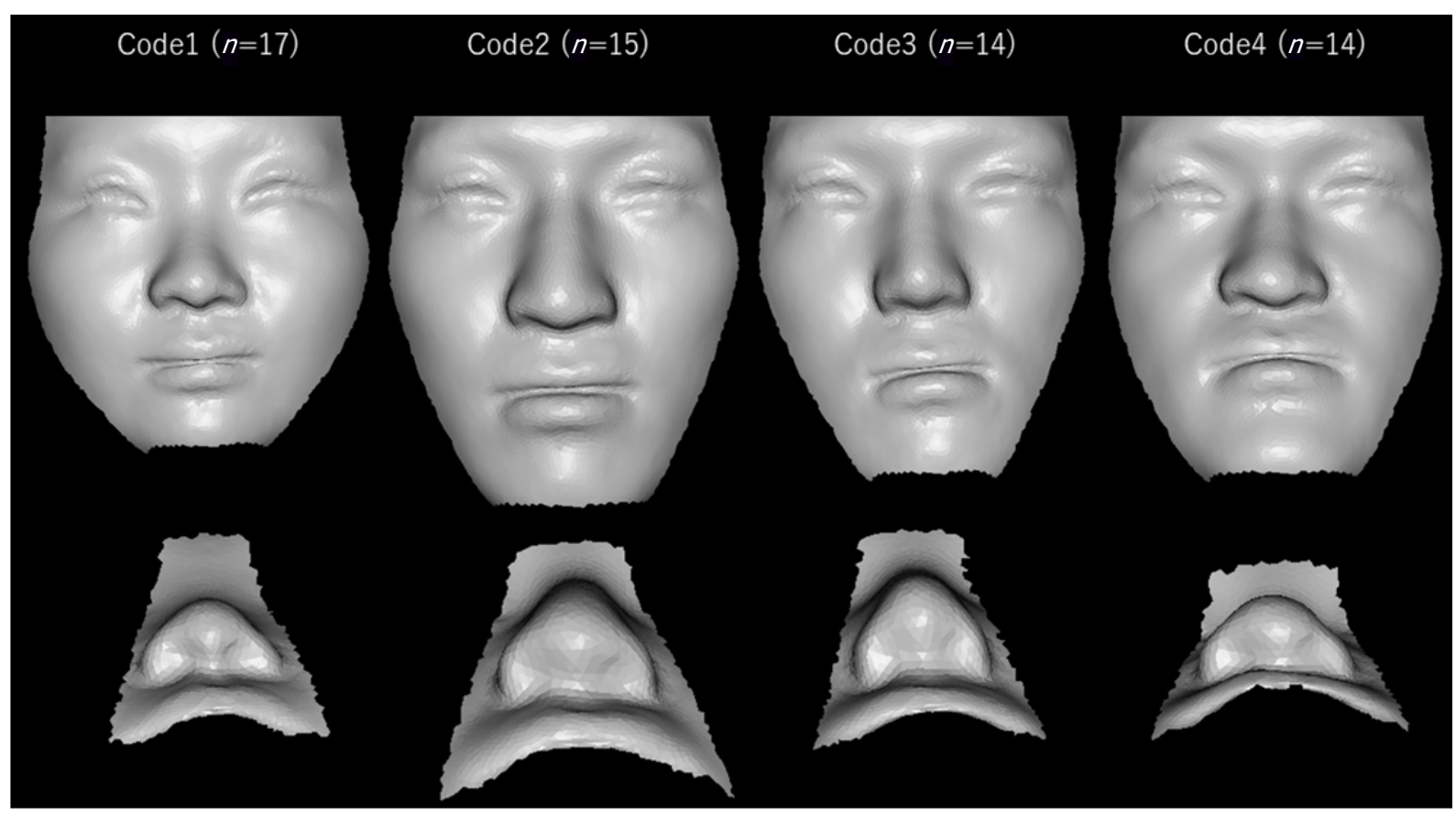

Figure 11. The results of the clustering method. Sixty samples were classified into four categories (codes). Figures 11 and 12 show that Code 1 represents patients having midface retrusion with short mandibular height; Code 2 represents those with midface retrusion combined with mandibular protrusion with well-developed nasal bone; Code 3 represents those with smaller nasal height, retruded cheeks, and asymmetric face; and Code 4 represents those with severe mandibular protrusion with asymmetric nose

applied to the face of one example patient with cleft lip and palate. GMM is considered to be effective for describing the changes from before to after surgery.

A key concept of GMM is based on the fact that morphology can be mapped in the same dimensions as space, i.e., "the morphospace", using these landmarks. Once 3D face images are converted to the morphospace, we can apply several statistical analyses to these face images. Examples of applications of GMM include the detection of facial sexual dimorphism ${ }^{[21]}$, the examination of relationships between faces and genetics ${ }^{[23,24]}$, the clinical diagnosis of dysmorphology ${ }^{[25]}$, computer vision ${ }^{[26]}$, and computer graphics ${ }^{[27]}$. In the present study, we used GMM for the quantitative evaluation of the treatment effects in patients with CLP and for the examination of the variation of the faces of patients with CLP based on a combination of principal component regression, MANOVA, and the clustering method (described below).

Quantitative evaluation of the treatment effect in patients with UCLP

GMM could be applied to detect the normal area before and after surgery. For the selected case, the percentage of the normal area was increased for all axes; however, several portions showing deformities 

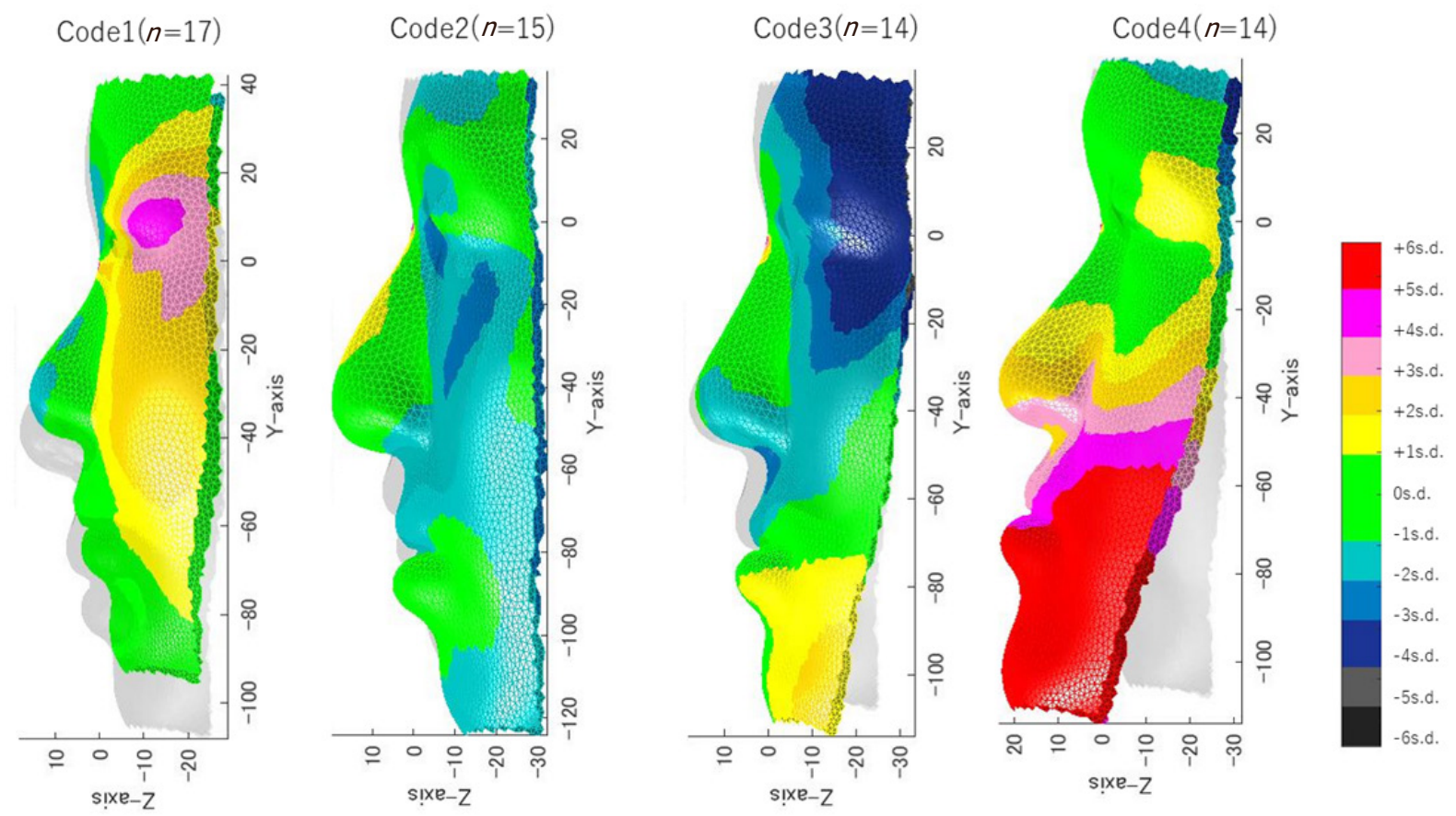

Figure 12. Z-scores for the four categories (Code) of Cleft patients. The average shape was evaluated with the normative range ${ }^{[19]}$. Only the $z$-axis (anterior-posterior direction) results were exemplified. A grey profile indicates the average face. $Z$-score was calculated by the equation: $Z$-score $(z)=\left(p_{(z)}-m_{(z)}\right) / s_{(z)}$ where $p_{(z)}$ indicates the averaged coordinate values of each code, $m_{(z)}$ indicates the average coordinate values of the control group, and $s_{(z)}$ indicates the standard deviation of the coordinate values of the control group in the z-direction. Please note that Code 1 shows that the anterior-posterior position of the upper and lower lips was within 1 SD. In Codes 2 and 3 , the cheeks (midface) were remarkably retruded, while the anterior-posterior position of the upper lip was within 1 SD. Codes 4 shows greater protrusion of upper and lower lips

\section{Table 2. Demographic data in each code}

\begin{tabular}{|c|c|c|c|c|}
\hline & Code 1 & Code 2 & Code 3 & Code 4 \\
\hline Patient number & 17 & 15 & 14 & 14 \\
\hline \multicolumn{5}{|l|}{ Sex } \\
\hline Male & 1 & 13 & 11 & 11 \\
\hline Female & 16 & 2 & 3 & 3 \\
\hline \multicolumn{5}{|l|}{ Cleft side } \\
\hline Left & 11 & 13 & 11 & 11 \\
\hline Right & 6 & 2 & 3 & 3 \\
\hline \multicolumn{5}{|l|}{ Orthodontic treatment } \\
\hline Camouflage & 4 & 4 & 2 & 3 \\
\hline Surgery & 13 & 11 & 12 & 11 \\
\hline \multicolumn{5}{|l|}{ Surgical technique for the palatoplasty } \\
\hline Wardill-Kilner push-back & 15 & 15 & 12 & 12 \\
\hline Early two-stage with modified Furlow veloplasty & 2 & 0 & 2 & 2 \\
\hline \multicolumn{5}{|l|}{ Age (years old) } \\
\hline Mean & 20.6 & 21.9 & 22.2 & 23.5 \\
\hline Standard deviation & 3.2 & 2.3 & 7.5 & 5.6 \\
\hline
\end{tabular}

remained, even after surgery. This was considered to be due to treatment limitations. Another possibility is that there were no measures to analyze the $3 \mathrm{D}$ face when we had performed surgery. The method introduced in the present article helps both patients and orthodontists share a mutual understanding of the soft tissue problems of patients with CLP. The patient's facial soft tissues can be quantified and visualized three-dimensionally. This is useful for developing optimum treatment plans based on the evaluation of soft tissue. 
Examination of the variation of the faces of patients with CLP

In the present study, we also clarified the morphological variations and characteristics of the face in patients with CLP using several statistical methods. This became possible because the faces could be converted to vectors of the same dimension (i.e., morphospace). Because the present study only included patients with positive overjet (i.e., most patients were in the post-treatment "retention" period), our study mainly detected antero-posterior facial deformities. If we examined patients before or during treatment, our method would be able to detect transverse facial deformities as well.

Decreased nasal height and its related obscure nasal tip were identified as characteristics of the Cleft group in the present study. These results were compatible with those of previous reports ${ }^{[6,28-30]}$. The Cleft group was also characterized by downward rotation of the dorsum and a well-developed nasal bone (PC3 and Code 2). This can be explained by the assumption that the retruded midface and deficient maxilla of the nose in patients resulted in a significant nasal hump and downward rotation of the columella. This observation was well-matched with a previous analysis from the lateral view ${ }^{[15]}$, which stated that this "beaky type nose" was often observed in adult patients with a cleft lip ${ }^{[15,29]}$.

A MANOVA also showed that the facial morphology was significantly different between the Cleft and Control groups and sex subgroups, and that the effects of a cleft on the facial morphology were related to sex. Figure 10 shows that patients in the Cleft group tended to have greater lower lip protrusion, and this was exaggerated in males. As our samples were from patients who underwent treatment in our hospital, this fact might be related to the treatment goal or treatment demands in males in our hospital.

\section{The $3 D$ analysis of patients with CLP}

The reason $3 \mathrm{D}$ technology has not shown any further clinical progress thus far can be due to technical challenges in the analysis of $3 \mathrm{D}$ structures ${ }^{[19]}$. To solve the problem that $3 \mathrm{D}$ images containing an enormous quantity of information cannot be fully utilized in GMMs, this study introduced a new method to statistically analyze the entire $3 \mathrm{D}$ morphology and to simply visualize the results in patients with CLP. For the treatment of patients with CLP, a 3D quantitative analysis of the craniofacial structures is important for determining the treatment objectives and results.

\section{Limitations}

There are two major limitations in this study that could be addressed in future research. First, the sample size for the exemplification of the system application was limited. Secondly, the specific features and categories identified in this study might be different from those observed in other centers.

\section{Conclusion}

GMM was applied for quantifying and visualizing the $3 \mathrm{D}$ configuration of the soft tissues of the face. This method was effective in describing the changes from before to after surgery. GMM was also used to detect morphological variations of the face in patients with CLP. GMM will be a powerful tool to instantaneously and comprehensively evaluate faces in clinics.

\section{DECLARATIONS}

\section{Authors' contributions}

The author contributed solely to the article.

\section{Availability of data and materials}

Not applicable. 


\section{Financial support and sponsorship}

This work was supported by JSPS KAKENHI (No. JP 19K10403). The funding body has no roles in the experiment design, collection, analysis and interpretation of data, and writing of the manuscript.

\section{Conflicts of interest}

The author declared that there are no conflicts of interest.

\section{Ethical approval and consent to participate}

Research involving human subjects, human material or human data was performed in accordance with the Declaration of Helsinki and approved by the ethics committee for medical research at Osaka University Dental Hospital (project ID: H25-E37-1). An informed consent to participate in the study was obtained from participants.

\section{Consent for publication}

A statement that a written informed consent for publication was obtained.

\section{Copyright}

(c) The Author(s) 2020.

\section{REFERENCES}

1. Sykes JM, Jang YJ. Cleft lip rhinoplasty. Facial Plast Surf Clin North Am 2009;17:133-44.

2. Yamada T, Mori Y, Minami K, Mishima K, Sugahara T. Three-dimensional facial morphology, following primary cleft lip repair using the triangular flap with or without rotation advancement. J Craniomaxillofac Surg 2002;30:337-42.

3. Fujimoto T, Imai K, Hatano T, Takahashi M, Tamai M. Follow-up of unilateral cleft-lip nose deformity after secondary repair with a modified reverse-U method. J Plast Reconstr Aesthet Surg 2011;64:747-53.

4. Mori A, Nakajima T, Kaneko T, Sakuma H, Aoki Y. Analysis of 109 Japanese children's lip and nose shapes using 3-dimensional digitizer. Br J Plast Surg 2005;58:318-29.

5. Anastassov Y, Chipkov C. Analysis of nasal and labial deformities in cleft lip, alveolus and palate patients by a new rating scale: preliminary report. J Craniomaxillofac Surg 2003;31:299-303.

6. Kim DW, Kim JT, Hong HK, Nam KC, Park JH. Statistical evaluation of the cleft lip nose deformity image. Conf Proc IEEE Eng Med Biol Soc 2006;1:3840-2.

7. Nagy K, Mommaerts MY. Analysis of the cleft-lip nose in submental-vertical view, Part I--reliability of a new measurement instrument. J Craniomaxillofac Surg 2007;35:265-77.

8. Farkas LG. Anthropometry of the head and face. 2nd ed. New York: Raven Press; 1994.

9. Ferrario VF, Sforza C, Dellavia C, Vizzotto L, Carù A. Three-dimensional nasal morphology in cleft lip and palate operated adult patients. Ann Plast Surg 2003;51:390-7.

10. Ras F, Habets LL, van Ginkel FC, Prahl-Andersen B. Method for quantifying facial asymmetry in three dimensions using stereophotogrammetry. Angle Orthod 1995;65:233-9.

11. van Loon B, Maal TJ, Plooij JM, Ingels KJ, Borstlap WA, et al. 3D Stereophotogrammetric assessment of pre- and postoperative volumetric changes in the cleft lip and palate nose. Int J Oral Maxillofac Surg 2010;39:534-40.

12. Russell KA, Waldman SD, Tompson B, Lee JM. Nasal morphology and shape parameters as predictors of nasal esthetics in individuals with complete unilateral cleft lip and palate. Cleft Palate Craniofac J 2001;38:476-85.

13. Russell KA, Milne AD, Varma D, Josephson K, Lee JM. Three-dimensional morphologic nasal surface characteristics that predict the extremes of esthetics in patients with repaired cleft lip and palate. Cleft Palate Craniofac J 2011;48:28-37.

14. Dusková M, Kristen M, Smahel Z. The anthropometric verification of corrective surgery outcome in cleft secondary deformities. J Craniofac Surg 2006;17:447-53.

15. Nagasao T, Miyamoto J, Hikosaka M, Yoshikawa K, Ishii N, et al. A new method to quantify subtle morphological deformities in nasal profile curvatures and its application for analysis of unilateral cleft lip noses. J Craniomaxillofac Surg 2008;36:321-34.

16. Yamada T, Mori Y, Minami K, Mishima K, Sugahara T, et al. Computer aided three-dimensional analysis of nostril forms: application in normal and operated cleft lip patients. J Craniomaxillofac Surg 1999;27:345-53.

17. Tanikawa C, Takada K, van Aalst J, Trotman CA. Objective three-dimensional assessment of lip form in patients with repaired cleft lip. Cleft Palate Craniofac J 2010;47:611-22.

18. Bookstein FL. Landmark methods for forms without landmarks: morphometrics of group differences in outline shape. Med Image Anal 1997; 1:225-43.

19. Tanikawa C, Akcam MO, Takada K. Quantifying faces three-dimensionally in orthodontic practice. J Cranio-maxillofacial Surgery 
2019;47:867-75.

20. Shintaku Y, Tanikawa C, Iida S, Aikawa T, Kogo M, et al. Maxillary expansion and midline correction by asymmetric transverse distraction osteogenesis in a patient with unilateral cleft lip/palate: a case report. Cleft Palate Craniofac J 2015;52:618-24.

21. Tanikawa C, Zere E, Takada K. Sexual dimorphism in the facial morphology of adult humans: a three-dimensional analysis. Homo 2016;67:23-49.

22. Kono K, Tanikawa C, Yanagita T, Kamioka H, Yamashiro T. A novel method to detect 3D mandibular changes related to soft-diet feeding. Front Physiol 2017;8:567.

23. Crouch DJM, Winney B, Koppen WP, Christmas WJ, Hutnik K, et al. Genetics of the human face: identification of large-effect single gene variants. Proc Natl Acad Sci U S A 2018;115:E676-85.

24. Claes P, Liberton DK, Daniels K, Rosana KM, Quillen EE, et al. Modeling 3D facial shape from DNA. Proc Natl Acad Sci USA 2018;115:E676-85.

25. Hammond P. The use of 3D face shape modelling in dysmorphology. Arch Dis Child 2007;92:1120-6.

26. Huber P, Hu G, Tena1 R, Mortazavian P, Koppen WP, et al. A multiresolution 3D morphable face model and fitting framework. Available from: http://www.ee.surrey.ac.uk/CVSSP/Publications/papers/Huber-VISAPP-2016.pdf. [Last accessed on 7 Sep 2020]

27. Yang H, Zhu H, Wang Y, Huang M, Shen Q, et al. FaceScape: a large-scale high quality 3D face Dataset and detailed riggable 3D face prediction. Available from: https://arxiv.org/abs/2003.13989. [Last accessed on 25 Aug 2020]

28. Farkas LG, Hajnis K, Posnick JC. Anthropometric and anthroposcopic findings of the nasal and facial region in cleft patients before and after primary lip and palate repair. Cleft Palate Craniofac J 1993;30:1-12.

29. Bardach J, Mooney MP. The relationship between lip pressure following lip repair and craniofacial growth: an experimental study in beagles. Plast Reconstr Surg 1984;73:544-55.

30. Ayoub A, Garrahy A, Millett D, Bowman A, Siebert P, et al. Three-dimensional assessment of early surgical outcome in repaired unilateral cleft lip and palate: Part 1. Nasal changes. Cleft Palate Craniofac J 2011;48:571-7. 